\title{
Energy levels and radiative rates for transitions in $\mathrm{Ni} \mathrm{XIX}^{\star}$
}

\author{
K. M. Aggarwal and F. P. Keenan
}

\begin{abstract}
Department of Physics and Astronomy, Queen's University, Belfast BT7 1NN, Northern Ireland, UK e-mail: K. Aggarwal@qub.ac.uk
\end{abstract}

Received 30 June 2006 / Accepted 13 September 2006

\section{ABSTRACT}

\begin{abstract}
Aims. In this paper we report calculations for energy levels and radiative rates for transitions in Ni XIX. Methods. The General purpose Relativistic Atomic Structure Package (GRASP) has been adopted for the calculation of energy levels and radiative rates.

Results. Energies for the lowest 89 levels from the $\left(1 \mathrm{~s}^{2}\right) 2 \mathrm{~s}^{2} 2 \mathrm{p}^{6}, 2 \mathrm{~s}^{2} 2 \mathrm{p}^{5} 3 \ell, 2 \mathrm{~s} 2 \mathrm{p}^{6} 3 \ell, 2 \mathrm{~s}^{2} 2 \mathrm{p}^{5} 4 \ell$ and $2 \mathrm{~s} 2 \mathrm{p}^{6} 4 \ell$ configurations of Ni XIX, are reported. Additionally, radiative rates, oscillator strengths, and line strengths are reported for all electric dipole (E1), magnetic dipole (M1), electric quadrupole (E2), and magnetic quadrupole (M2) transitions among these levels. Comparisons are made with the available results in the literature, and the accuracy of the present data is assessed. Finally, lifetimes for all excited levels are listed, and comparisons made with earlier available theoretical results.
\end{abstract}

Key words. atomic data - atomic processes

\section{Introduction}

Neon-like ions, particularly of the iron group of elements (namely $\mathrm{Ti}, \mathrm{Cr}, \mathrm{Fe}$ and $\mathrm{Ni}$ ), are highly useful for the modelling and diagnostics of a variety of plasmas, such as astrophysical, fusion and laser generated plasmas. Many emission lines of nickel, including those from Ni XIX, have been observed in the sun - see, for example, Jupen (1984) and Feldman et al. (2000). Similarly many transitions, particularly within the $n=3$ configurations, have been measured in laboratory plasmas by Feldman et al. (1967), Swartz et al. (1971), Boiko et al. (1977), Buchet et al. (1987) and Biémont et al. (2000). Therefore, atomic data (energy levels, radiative rates, collision strengths and excitation rates) for Ni XIX (and many other ions) are required for the modelling of plasmas, as well as diagnostics for their physical parameters, such as temperature and density. To fulfill this requirement, we have already reported results of energy levels and radiative rates for transitions in Ni XIII-XVI (Aggarwal et al. 2003) and Ni XVII (Aggarwal et al. 2007), and in this paper we present similar results for Ni XIX.

There have been a few calculations in the literature for Ni XIX, particularly by Loulergue \& Nussbaumer (1975), Zhang et al. (1987), Zhang \& Sampson (1989), and Hibbert et al. (1993). Loulergue \& Nussbaumer reported values of energy levels and radiative rates ( $A$-values). For the construction of wavefunctions, they included configuration interaction (CI) among 89 levels of the $\left(1 \mathrm{~s}^{2}\right) 2 \mathrm{~s}^{2} 2 \mathrm{p}^{6}, 2 \mathrm{~s}^{2} 2 \mathrm{p}^{5} 3 \ell, 2 \mathrm{~s} 2 \mathrm{p}^{6} 3 \ell$, $2 \mathrm{~s}^{2} 2 \mathrm{p}^{5} 4 \ell$ and $2 \mathrm{~s} 2 \mathrm{p}^{6} 4 \ell$ configurations, but reported energy levels and $A$-values only for some transitions among 57 levels. Furthermore, they calculated $A$-values for the electric dipole (E1) transitions alone, whereas in plasma modelling corresponding results for other types of transitions, namely magnetic dipole (M1), electric quadrupole (E2), and magnetic quadrupole (M2), may also be required. Similarly,

* Tables 3 and 4 are available only in electronic form at the CDS via anonymous ftp to cdsarc.u-strasbg.fr (130.79.128.5) or via http://cdsweb.u-strasbg.fr/cgi-bin/qcat? J/A+A/460/959
Zhang et al. and Zhang \& Sampson also calculated $A$-values, but for resonance E1 transitions alone. Finally, Hibbert et al. adopted the semi-relativistic CIV3 program (Hibbert 1975a) in which they included extensive CI with up to $n=5$ orbitals. However, their calculations are restricted to E1 transitions among the lowest 37 levels of the $2 s^{2} 2 p^{6}, 2 s^{2} 2 p^{5} 3 \ell$ and $2 s^{2} p^{6} 3 \ell$ configurations, whereas emission lines from the higher excited levels of the $n=4$ and 5 configurations have already been measured (Swartz et al. 1971; Boiko et al. 1977; Biémont et al. 2000). Therefore, there is a clear need to extend the calculations to higher excited levels. Additionally, and more importantly, their energy levels, particularly for the levels of the $2 \mathrm{~s} 2 \mathrm{p}^{6} 3 \ell$ configurations, differ by up to $\sim 1.6$ Ryd (2\%) with those of Zhang \& Sampson or the experimental values. Therefore, it has become essential to resolve this large discrepancy, apart from extending their calculations to higher excited levels, so that the results can be applied with confidence to the modelling of plasmas. Hence, in this paper we report energy levels and radiative rates for all transitions, and for all the four types mentioned above, because both of these requirements of having a complete set of data and for all types of transitions have recently been emphasized by Liedahl (2000), and demonstrated by Del Zanna et al. (2004).

In the present work, we mostly focus on the lowest 89 levels of the $2 s^{2} 2 p^{6}, 2 s^{2} 2 p^{5} 3 \ell, 2 s 2 p^{6} 3 \ell, 2 s^{2} 2 p^{5} 4 \ell$ and $2 s^{2} 2 p^{6} 4 \ell$ configurations of Ni XIX, although calculations have been performed for a larger number of levels (157). Additionally, our approach is fully relativistic, as we employ the GRASP (General purpose Relativistic Atomic Structure Package) code of Dyall et al. (1989) to calculate radiative rates for the four types of transitions mentioned above. Finally, we also report lifetimes for all excited levels, and make comparisons with other available theoretical results.

\section{Energy levels}

The $2 s^{2} 2 p^{6}, 2 s^{2} 2 p^{5} 3 \ell, 2 s 2 p^{6} 3 \ell, 2 s^{2} 2 p^{5} 4 \ell$ and $2 s 2 p^{6} 4 \ell$ configurations of Ni XIX give rise to 89 fine-structure levels, listed in 
Table 1. Target levels of Ni XIX, their threshold energies (in Ryd) and lifetimes ( $\tau$ in s). $a \pm b \equiv a \times 10^{ \pm b}$.

\begin{tabular}{|c|c|c|c|c|c|c|c|c|c|c|}
\hline Index & Configuration & Level & Expt. & $\mathrm{LN}$ & $\mathrm{ZS}$ & $\operatorname{GRASP}^{a}$ & GRASP $^{b}$ & GRASP $^{c}$ & $\tau^{d}$ & $\tau^{e}$ \\
\hline 1 & $2 s^{2} 2 p^{6}$ & ${ }^{1} \mathrm{~S}_{0}$ & 0.00000 & 0.000 & 0.0000 & 0.00000 & 0.00000 & 0.00000 & & \\
\hline 2 & $2 s^{2} 2 p^{5} 3 s$ & ${ }^{3} \mathrm{P}_{2}^{\circ}$ & 64.74789 & 64.651 & 64.7262 & 64.65407 & 64.59266 & 64.60110 & $2.108-06$ & $2.125-06$ \\
\hline 3 & $2 s^{2} 2 p^{5} 3 s$ & ${ }^{1} \mathrm{P}_{1}^{\circ}$ & 64.90591 & 66.026 & 66.1669 & 64.81635 & 64.75556 & 64.76398 & $6.851-13$ & $6.886-13$ \\
\hline 4 & $2 s^{2} 2 p^{5} 3 s$ & ${ }^{3} \mathrm{P}_{0}^{\circ}$ & 66.04590 & 65.925 & 66.0566 & 65.98467 & 65.89549 & 65.90393 & $2.205-05$ & $2.205-05$ \\
\hline 5 & $2 s^{2} 2 p^{5} 3 s$ & ${ }^{3} \mathrm{P}_{1}^{\circ}$ & 66.14067 & 64.813 & 64.8953 & 66.08407 & 65.99248 & 66.00089 & $8.639-13$ & $8.687-13$ \\
\hline 6 & $2 s^{2} 2 p^{5} 3 p$ & ${ }^{3} S_{1}$ & 67.26964 & 66.892 & 67.2400 & 67.17989 & 67.11863 & 67.12572 & $2.319-10$ & $2.327-10$ \\
\hline 7 & $2 s^{2} 2 p^{5} 3 p$ & ${ }^{3} \mathrm{D}_{2}$ & 67.52411 & 67.165 & 67.5119 & 67.44378 & 67.38277 & 67.39132 & $1.456-10$ & $1.458-10$ \\
\hline 8 & $2 s^{2} 2 p^{5} 3 p$ & ${ }^{3} \mathrm{D}_{3}$ & 67.72295 & 67.368 & 67.7178 & 67.64860 & 67.57916 & 67.58763 & $1.262-10$ & $1.263-10$ \\
\hline 9 & $2 s^{2} 2 p^{5} 3 p$ & ${ }^{1} \mathrm{P}_{1}$ & 67.79872 & 68.395 & 67.7913 & 67.72326 & 67.65968 & 67.66821 & $1.376-10$ & $1.375-10$ \\
\hline 10 & $2 s^{2} 2 p^{5} 3 p$ & ${ }^{3} \mathrm{P}_{2}$ & 67.96467 & 67.608 & 67.9603 & 67.88865 & 67.82370 & 67.83239 & $9.417-11$ & $9.424-11$ \\
\hline 11 & $2 s^{2} 2 p^{5} 3 p$ & ${ }^{3} \mathrm{P}_{0}$ & 68.48787 & 68.138 & 68.5042 & 68.43553 & 68.36453 & 68.37167 & $8.498-11$ & $8.480-11$ \\
\hline 12 & $2 s^{2} 2 p^{5} 3 p$ & ${ }^{3} \mathrm{D}_{1}$ & 68.77114 & 68.720 & 68.7982 & 68.72702 & 68.63561 & 68.64413 & $1.757-10$ & $1.756-10$ \\
\hline 13 & $2 s^{2} 2 p^{5} 3 p$ & ${ }^{3} \mathrm{P}_{1}$ & 69.10029 & 67.442 & 69.1216 & 69.05203 & 68.95945 & 68.96780 & $1.119-10$ & $1.119-10$ \\
\hline 14 & $2 s^{2} 2 p^{5} 3 p$ & ${ }^{1} \mathrm{D}_{2}$ & 69.14025 & 69.040 & 69.1731 & 69.09959 & 69.00116 & 69.00972 & $1.049-10$ & $1.051-10$ \\
\hline 15 & $2 s^{2} 2 p^{5} 3 p$ & ${ }^{1} \mathrm{~S}_{0}$ & 70.08373 & 69.883 & 70.2829 & 70.20187 & 70.13098 & 70.12033 & $2.980-11$ & 3.014-11 \\
\hline 16 & $2 s^{2} 2 p^{5} 3 d$ & ${ }^{3} \mathrm{P}_{0}^{\circ}$ & 71.06029 & 70.530 & 71.0474 & 70.97559 & 70.91200 & 70.91987 & 7.318-11 & $7.335-11$ \\
\hline 17 & $2 s^{2} 2 p^{5} 3 d$ & ${ }^{3} \mathrm{P}_{1}^{0}$ & 71.14900 & 70.626 & 71.1430 & 71.07218 & 71.00144 & 71.00934 & $6.579-12$ & $6.564-12$ \\
\hline 18 & $2 s^{2} 2 p^{5} 3 d$ & ${ }^{3} \mathrm{P}_{2}^{1}$ & 71.31366 & 70.804 & 71.3194 & 71.24548 & 71.16951 & 71.17763 & $7.524-11$ & $7.536-11$ \\
\hline 19 & $2 s^{2} 2 p^{5} 3 d$ & ${ }^{3} \mathrm{~F}_{4}^{\circ}$ & 71.30920 & 70.816 & 71.3194 & 71.24872 & 71.16811 & 71.17517 & 7.866-11 & $7.887-11$ \\
\hline 20 & $2 s^{2} 2 p^{5} 3 d$ & ${ }^{3} \mathrm{~F}_{3}^{\circ}$ & 71.36068 & 70.860 & 71.3782 & 71.30334 & 71.22664 & 71.23402 & $6.555-11$ & $6.565-11$ \\
\hline 21 & $2 s^{2} 2 p^{5} 3 d$ & ${ }^{1} \mathrm{D}_{2}^{\circ}$ & 71.50804 & 72.206 & 71.5252 & 71.44977 & 71.37793 & 71.38611 & $6.317-11$ & $6.320-11$ \\
\hline 22 & $2 s^{2} 2 p^{5} 3 d$ & ${ }^{3} \mathrm{D}_{3}^{\circ}$ & 71.60408 & 71.120 & 71.6281 & 71.55273 & 71.47633 & 71.48470 & $6.954-11$ & $6.953-11$ \\
\hline 23 & $2 s^{2} 2 p^{5} 3 d$ & ${ }^{3} \mathrm{D}_{1}^{\mathrm{o}}$ & 72.00285 & 71.550 & 72.0764 & 71.99795 & 71.91932 & 71.92557 & $8.806-14$ & 8.804-14 \\
\hline 24 & $2 s^{2} 2 p^{5} 3 d$ & ${ }^{3} \mathrm{~F}_{2}^{\circ}$ & 72.65054 & 72.122 & 72.7012 & 72.62254 & 72.51867 & 72.52627 & $6.377-11$ & $6.386-11$ \\
\hline 25 & $2 s^{2} 2 p^{5} 3 d$ & ${ }^{3} \mathrm{D}_{2}^{\circ}$ & 72.72649 & 71.008 & 72.7747 & 72.69682 & 72.59210 & 72.60051 & $7.158-11$ & $7.161-11$ \\
\hline 26 & $2 s^{2} 2 p^{5} 3 d$ & ${ }^{1} \mathrm{~F}_{3}^{\circ}$ & 72.77962 & 72.269 & 72.8335 & 72.75651 & 72.64864 & 72.65641 & $7.330-11$ & $7.335-11$ \\
\hline 27 & $2 s^{2} 2 p^{5} 3 d$ & ${ }^{1} \mathrm{P}_{1}^{\circ}$ & 73.28227 & 72.874 & 73.4436 & 73.35168 & 73.24505 & 73.24640 & $2.832-14$ & $2.874-14$ \\
\hline 28 & $2 s 2 p^{6} 3 s$ & ${ }^{3} S_{1}^{1}$ & 76.16370 & 74.822 & 76.0309 & 75.99472 & 75.91019 & 75.91787 & $5.375-12$ & $5.350-12$ \\
\hline 29 & $2 \mathrm{~s} 2 \mathrm{p}^{6} 3 \mathrm{~s}$ & ${ }^{1} \mathrm{~S}_{0}$ & 76.69223 & 75.364 & 76.5821 & 76.53857 & 76.45810 & 76.46122 & $7.771-12$ & $7.776-12$ \\
\hline 30 & $2 s 2 p^{6} 3 p$ & ${ }^{3} \mathrm{P}_{0}^{\circ}$ & & 77.249 & 78.7284 & 78.70060 & 78.62091 & 78.62883 & $5.817-12$ & $5.818-12$ \\
\hline 31 & $2 s^{2} p^{6} 3 p$ & ${ }^{3} \mathrm{P}_{1}^{\circ}$ & 78.56398 & 77.294 & 78.7725 & 78.74533 & 78.66211 & 78.67007 & $1.047-12$ & $1.052-12$ \\
\hline 32 & $2 s^{2} p^{6} 3 p$ & ${ }^{3} \mathrm{P}_{2}^{\circ}$ & & 77.552 & 79.0371 & 79.00541 & 78.91529 & 78.92313 & $5.701-12$ & $5.700-12$ \\
\hline 33 & $2 s^{2} p^{6} 3 p$ & ${ }^{1} \mathrm{P}_{1}^{\circ}$ & 78.97314 & 77.707 & 79.1915 & 79.15871 & 79.06836 & 79.07669 & $1.987-13$ & $1.994-13$ \\
\hline 34 & $2 \mathrm{~s}^{2} \mathrm{p}^{6} 3 \mathrm{~d}$ & ${ }^{3} \mathrm{D}_{1}$ & & 80.828 & 82.4844 & 82.45243 & 82.35964 & 82.36300 & $6.964-12$ & $7.091-12$ \\
\hline 35 & $2 s^{2} p^{6} 3 d$ & ${ }^{3} \mathrm{D}_{2}$ & & 80.852 & 82.4991 & 82.47276 & 82.37523 & 82.37867 & $6.825-12$ & $6.941-12$ \\
\hline 36 & $2 s^{2} p^{6} 3 d$ & ${ }^{3} \mathrm{D}_{3}$ & & 80.894 & 82.5358 & 82.50711 & 82.40539 & 82.40894 & $6.703-12$ & $6.810-12$ \\
\hline 37 & $2 s^{2} p^{6} 3 d$ & ${ }^{1} \mathrm{D}_{2}$ & & 81.316 & 82.9621 & 82.92779 & 82.82932 & 82.83494 & $5.643-12$ & 5.691-12 \\
\hline 38 & $2 s^{2} 2 p^{5} 4 s$ & ${ }^{3} \mathrm{P}_{2}^{\circ}$ & & 86.992 & 87.3576 & 87.25502 & 87.18001 & 87.18820 & $1.873-12$ & $1.862-12$ \\
\hline 39 & $2 s^{2} 2 p^{5} 4 s$ & ${ }^{1} \mathrm{P}_{1}^{\circ}$ & 87.34495 & 87.048 & 87.4090 & 87.30803 & 87.23366 & 87.24178 & $9.133-13$ & $9.365-13$ \\
\hline 40 & $2 s^{2} 2 p^{5} 4 p$ & ${ }^{3} S_{1}^{1}$ & & & 88.4087 & 88.30838 & 88.23420 & 88.24100 & $1.976-12$ & $1.953-12$ \\
\hline 41 & $2 s^{2} 2 p^{5} 4 p$ & ${ }^{3} \mathrm{D}_{2}$ & & & 88.6365 & 88.37214 & 88.29789 & 88.30644 & $1.907-12$ & $1.904-12$ \\
\hline 42 & $2 s^{2} 2 p^{5} 4 p$ & ${ }^{3} \mathrm{D}_{3}$ & & & 88.5630 & 88.45761 & 88.37966 & 88.38816 & $2.024-12$ & $2.007-12$ \\
\hline 43 & $2 s^{2} 2 p^{5} 4 p$ & ${ }^{1} \mathrm{P}_{1}$ & & & 88.5851 & 88.48478 & 88.40888 & 88.41725 & $1.901-12$ & $1.926-12$ \\
\hline 44 & $2 s^{2} 2 p^{5} 4 p$ & ${ }^{3} \mathrm{P}_{2}$ & & & 89.9375 & 88.53920 & 88.46323 & 88.47188 & $2.059-12$ & $2.060-12$ \\
\hline 45 & $2 s^{2} 2 p^{5} 4 s$ & $\begin{array}{l}{ }^{3} \mathrm{P}_{2}^{\circ} \\
\mathrm{P}_{0}\end{array}$ & & 88.269 & 88.7027 & 88.59220 & 88.48770 & 88.49586 & $1.868-12$ & $1.856-12$ \\
\hline 46 & $2 s^{2} 2 p^{5} 4 s$ & ${ }^{3} \mathrm{P}_{1}^{0}$ & 88.62072 & 88.297 & 88.7321 & 88.61951 & 88.51422 & 88.52234 & $1.060-12$ & $1.082-12$ \\
\hline 47 & $2 s^{2} 2 p^{5} 4 p$ & $\begin{array}{l}{ }^{1} \mathrm{P}_{0} \\
{ }^{2}\end{array}$ & & & 88.9746 & 88.88798 & 88.81253 & 88.81156 & $2.125-12$ & $2.182-12$ \\
\hline 48 & $2 s^{2} 2 p^{5} 4 p$ & ${ }^{3} \mathrm{D}_{1}$ & & & 89.7905 & 89.68797 & 89.58303 & 89.59146 & $1.865-12$ & $1.865-12$ \\
\hline 49 & $2 s^{2} 2 p^{5} 4 d$ & ${ }^{3} \mathrm{P}_{0}^{\circ}$ & & 89.265 & 89.8126 & 89.69949 & 89.62341 & 89.63083 & $1.128-12$ & $1.115-12$ \\
\hline 50 & $2 s^{2} 2 p^{5} 4 d$ & ${ }^{3} \mathrm{P}_{1}^{\circ}$ & 89.71424 & 89.306 & 89.8493 & 89.74025 & 89.66194 & 89.66951 & $1.040-12$ & $1.032-12$ \\
\hline 51 & $2 s^{2} 2 p^{5} 4 d$ & ${ }^{3} \mathrm{~F}_{4}^{\circ}$ & 89.87827 & 89.360 & 89.9008 & 89.79252 & 89.71042 & 89.71858 & $1.126-12$ & $1.118-12$ \\
\hline 52 & $2 s^{2} 2 p^{5} 4 d$ & ${ }^{3} \mathrm{P}_{2}^{\circ}$ & & 89.372 & 89.9155 & 89.80543 & 89.72552 & 89.73351 & $1.131-12$ & $1.124-12$ \\
\hline 53 & $2 s^{2} 2 p^{5} 4 d$ & ${ }^{3} \mathrm{~F}_{3}^{\circ}$ & 91.15951 & 90.698 & 89.9155 & 89.81022 & 89.72977 & 89.73812 & $1.137-12$ & $1.134-12$ \\
\hline 54 & $2 s^{2} 2 p^{5} 4 p$ & ${ }^{3} \mathrm{P}_{1}^{3}$ & & & 89.9228 & 89.81331 & 89.70737 & 89.71520 & $2.041-12$ & 2.034-12 \\
\hline 55 & $2 s^{2} 2 p^{5} 4 p$ & ${ }^{1} \mathrm{D}_{2}$ & & & 88.4748 & 89.83401 & 89.72619 & 89.73473 & $2.019-12$ & $2.015-12$ \\
\hline 56 & $2 s^{2} 2 p^{5} 4 d$ & ${ }^{1} \mathrm{D}_{2}^{\circ}$ & 91.18867 & 90.669 & 89.9669 & 89.86255 & 89.78376 & 89.79229 & $1.143-12$ & $1.144-12$ \\
\hline 57 & $2 s^{2} 2 p^{5} 4 d$ & ${ }^{3} \mathrm{D}_{3}^{\circ}$ & 89.89741 & 89.469 & 90.0037 & 89.89999 & 89.81961 & 89.82821 & $1.138-12$ & $1.141-12$ \\
\hline 58 & $2 s^{2} 2 p^{5} 4 p$ & ${ }^{1} \mathrm{~S}_{0}{ }^{3}$ & & & 90.1507 & 90.06286 & 89.96517 & 89.95715 & $2.058-12$ & $2.138-12$ \\
\hline 59 & $2 s^{2} 2 p^{5} 4 d$ & ${ }^{3} \mathrm{D}_{1}^{\circ}$ & 90.13342 & 89.703 & 90.2242 & 90.13488 & 90.05354 & 90.05585 & $9.439-14$ & $9.926-14$ \\
\hline 60 & $2 s^{2} 2 p^{5} 4 f$ & ${ }^{3} \mathrm{D}_{1}$ & & & 90.5696 & 90.45328 & 90.37202 & 90.38075 & $4.825-13$ & $4.810-13$ \\
\hline 61 & $2 s^{2} 2 p^{5} 4 f$ & ${ }^{1} \mathrm{G}_{4}$ & & & 90.5770 & 90.46263 & 90.37912 & 90.38694 & $5.116-13$ & $5.110-13$ \\
\hline 62 & $2 s^{2} 2 p^{5} 4 f$ & ${ }^{3} \mathrm{G}_{5}$ & & & 90.5843 & 90.46612 & 90.38262 & 90.39042 & $5.105-13$ & $5.092-13$ \\
\hline 63 & $2 s^{2} 2 p^{5} 4 f$ & ${ }^{3} \mathrm{D}_{2}$ & & & 90.5843 & 90.46836 & 90.38653 & 90.39524 & $4.901-13$ & $4.896-13$ \\
\hline
\end{tabular}


Table 1. continued.

\begin{tabular}{|c|c|c|c|c|c|c|c|c|c|c|}
\hline Index & Configuration & Level & Expt. & $\mathrm{LN}$ & $\mathrm{ZS}$ & GRASP $^{a}$ & GRASP $^{b}$ & GRASP $^{c}$ & $\tau^{d}$ & $\tau^{e}$ \\
\hline 64 & $2 s^{2} 2 p^{5} 4 f$ & ${ }^{3} \mathrm{~F}_{3}$ & & & 90.6137 & 90.50041 & 90.41809 & 90.42677 & $4.978-13$ & $4.980-13$ \\
\hline 65 & $2 s^{2} 2 p^{5} 4 f$ & ${ }^{1} \mathrm{D}_{2}$ & & & 90.6211 & 90.50525 & 90.42322 & 90.43188 & $5.119-13$ & $5.146-13$ \\
\hline 66 & $2 s^{2} 2 p^{5} 4 f$ & ${ }^{1} \mathrm{~F}_{3}$ & & & 90.6211 & 90.50969 & 90.42724 & 90.43570 & $5.088-13$ & $5.095-13$ \\
\hline 67 & $2 s^{2} 2 p^{5} 4 f$ & ${ }^{3} \mathrm{~F}_{4}$ & & & 90.6358 & 90.51836 & 90.43562 & 90.44406 & $5.108-13$ & $5.118-13$ \\
\hline 68 & $2 s^{2} 2 p^{5} 4 d$ & ${ }^{3} \mathrm{~F}_{2}^{\circ}$ & & 90.640 & 91.2459 & 91.13541 & 91.02549 & 91.03375 & $1.140-12$ & $1.134-12$ \\
\hline 69 & $2 s^{2} 2 p^{5} 4 d$ & ${ }^{3} \mathrm{D}_{2}^{\circ}$ & & 89.428 & 91.2753 & 91.16235 & 91.05187 & 91.05988 & $1.132-12$ & $1.127-12$ \\
\hline 70 & $2 s^{2} 2 p^{5} 4 d$ & ${ }^{1} \mathrm{~F}_{3}^{\circ}$ & & 89.376 & 91.2973 & 91.18985 & 91.07845 & 91.08682 & $1.131-12$ & $1.129-12$ \\
\hline 71 & $2 s^{2} 2 p^{5} 4 d$ & ${ }^{1} \mathrm{P}_{1}^{\circ}$ & 91.33630 & 90.876 & 91.4664 & 91.36706 & 91.25686 & 91.25816 & $8.929-14$ & $9.644-14$ \\
\hline 72 & $2 s^{2} 2 p^{5} 4 f$ & ${ }^{3} \mathrm{G}_{3}$ & & & 91.9368 & 91.81666 & 91.70349 & 91.71148 & $5.093-13$ & $5.088-13$ \\
\hline 73 & $2 s^{2} 2 p^{5} 4 f$ & ${ }^{3} \mathrm{G}_{4}$ & & & 91.9515 & 91.82875 & 91.71542 & 91.72342 & $5.117-13$ & $5.116-13$ \\
\hline 74 & $2 s^{2} 2 p^{5} 4 f$ & ${ }^{3} \mathrm{~F}_{2}$ & & & 91.9515 & 91.83513 & 91.72231 & 91.73097 & $5.062-13$ & $5.077-13$ \\
\hline 75 & $2 s^{2} 2 p^{5} 4 f$ & ${ }^{3} \mathrm{D}_{3}$ & & & 91.9588 & 91.83544 & 91.72243 & 91.73112 & $4.974-13$ & $4.972-13$ \\
\hline 76 & $2 s-p^{6} 4 s$ & ${ }^{3} \mathrm{~S}_{1}$ & & & 98.5079 & 98.44633 & 98.34835 & 98.36363 & $1.375-12$ & $1.445-12$ \\
\hline 77 & $2 s 2 p^{6} 4 s$ & ${ }^{1} \mathrm{~S}_{0}$ & & & 98.6917 & 98.63700 & 98.54064 & 98.54400 & $1.440-12$ & $1.484-12$ \\
\hline 78 & $2 s 2 p^{6} 4 p$ & ${ }^{3} \mathrm{P}_{0}^{\circ}$ & & 97.992 & 99.6031 & 99.54575 & 99.45014 & 99.47090 & $1.452-12$ & $1.505-12$ \\
\hline 79 & $2 s 2 p^{6} 4 p$ & ${ }^{3} \mathrm{P}_{1}^{0}$ & 99.55593 & 98.008 & 99.6178 & 99.56155 & 99.46456 & 99.47209 & $7.905-13$ & $3.700-13$ \\
\hline 80 & $2 s 2 p^{6} 4 p$ & ${ }^{3} \mathrm{P}_{2}^{\circ}$ & & 98.115 & 99.7207 & 99.66750 & 99.56767 & 99.60480 & $1.516-12$ & $1.661-12$ \\
\hline 81 & $2 s^{2} p^{6} 4 p$ & ${ }^{1} \mathrm{P}_{1}^{\circ}$ & 99.70173 & 98.165 & 99.7721 & 99.71890 & 99.61892 & 99.59995 & $2.755-13$ & $1.754-13$ \\
\hline 82 & $2 \mathrm{~s} 2 \mathrm{p}^{6} 4 \mathrm{~d}$ & ${ }^{3} \mathrm{D}_{1}$ & & & 101.0217 & 100.96287 & 100.86169 & 100.87703 & $9.643-13$ & $9.533-13$ \\
\hline 83 & $2 \mathrm{~s} 2 \mathrm{p}^{6} 4 \mathrm{~d}$ & ${ }^{3} \mathrm{D}_{2}$ & & & 101.0290 & 100.97161 & 100.86875 & 100.88480 & $9.606-13$ & $9.473-13$ \\
\hline 84 & $2 \mathrm{~s} 2 \mathrm{p}^{6} 4 \mathrm{~d}$ & ${ }^{3} \mathrm{D}_{3}$ & & & 101.0511 & 100.98691 & 100.88248 & 100.89953 & $9.546-13$ & $9.383-13$ \\
\hline 85 & $2 \mathrm{~s} 2 \mathrm{p}^{6} 4 \mathrm{~d}$ & ${ }^{1} \mathrm{D}_{2}$ & & & 101.1907 & 101.13353 & 101.03066 & 101.04124 & $9.614-13$ & $9.748-13$ \\
\hline 86 & $2 s 2 p^{6} 4 f$ & ${ }^{3} \mathrm{~F}_{2}^{\circ}$ & & & 101.6979 & 101.62450 & 101.51947 & 101.53600 & $4.776-13$ & $4.724-13$ \\
\hline 87 & $2 s 2 p^{6} 4 f$ & ${ }^{3} \mathrm{~F}_{3}^{\circ}$ & & & 101.6979 & 101.62718 & 101.52125 & 101.54000 & $4.774-13$ & $4.726-13$ \\
\hline 88 & $2 \mathrm{~s} 2 \mathrm{p}^{6} 4 \mathrm{f}$ & ${ }^{3} \mathrm{~F}_{4}^{\circ}$ & & & 101.7053 & 101.63345 & 101.52723 & 101.54717 & $4.775-13$ & $4.729-13$ \\
\hline 89 & $2 \mathrm{~s} 2 \mathrm{p}^{6} 4 \mathrm{f}$ & ${ }^{1} \mathrm{~F}_{3}^{\circ}$ & & & 101.7200 & 101.64600 & 101.54040 & 101.55787 & 4.839-13 & 4.807-13 \\
\hline
\end{tabular}

Expt.: NIST data from http://www.physics.nist.gov/PhysRefData

LN: Loulergue \& Nussbaumer (1975).

ZS: Zhang \& Sampson (1989).

${ }^{a}$ Present GRASP results for 89 levels without Breit and QED effects.

${ }^{b}$ Present GRASP results for 89 levels with Breit and QED effects.

${ }^{c}$ Present GRASP results for 157 levels with Breit and QED effects.

${ }^{d}$ Present GRASP results for 89 levels.

${ }^{e}$ Present GRASP results for 157 levels.

Table 1. A calculation performed with these 15 configurations is referred to as GRASP1. Since some levels of the $n=5$ configurations interact with those of the $n=4$ configurations, we have performed another calculation, referred to as GRASP2, in which we include a total of 157 levels. The additional 68 levels arise from the $2 \mathrm{~s}^{2} 2 \mathrm{p}^{5} 5 \ell$ and $2 \mathrm{~s} 2 \mathrm{p}^{6} 5 \ell$ configurations. This will help us in assessing the accuracy of results for the lower levels.

Our calculated energies obtained from the GRASP code, from both the GRASP 1 and GRASP 2 calculations, are listed in Table 1 for the desired 89 levels. For our calculations, we have used the option of extended average level (EAL), in which a weighted (proportional to $2 j+1$ ) trace of the Hamiltonian matrix is minimized. This produces a compromise set of orbitals describing closely lying states with moderate accuracy. The calculations also include corrections from the Breit and QED effects. Also included in Table 1 are the experimental compilations of the National Institute of Standards and Technology (NIST), which are available at the website http://physics.nist.gov/PhysRefData, and the available theoretical results of Loulergue \& Nussbaumer (1975) and Zhang \& Sampson (1989).

Our energy levels from the GRASP1 and GRASP2 calculations agree closely (within 0.02 Ryd), except for the $2 \mathrm{~s} 2 \mathrm{p}^{6} 4 \mathrm{p}^{3} \mathrm{P}_{2}^{\circ}$ level (80), for which the difference is $0.04 \mathrm{Ryd}$. This indicates that the effect of the $n=5$ configurations is negligible on the energy levels of the $n=3$ and 4 configurations. This is mainly because the levels of the $n=5$ configurations lie above the $2 s^{2} 2 p^{5} 4 f^{3} D_{3}(75)$ level, and hence a majority of these do not interact with the lower levels. Additionally, level orderings are the same in both calculations. However, some levels, such as: $\left(2 s^{2} 2 p^{5} 3 p\right){ }^{3} D_{2}$ and ${ }^{1} D_{2}(7$ and 14$)$ and $\left(2 s^{2} 2 p^{5} 4 p\right){ }^{3} D_{2}$ and ${ }^{1} D_{2}$ (41 and 55), are highly (or rather equally) mixed. Nevertheless, we have identified these levels on the basis of the strengths of their eigenvectors, in both calculations, and can state with confidence that there is no uncertainty in the designations of the levels in our listings in Table 1.

Our Breit and QED corrected energy levels agree with the experimental values within 0.25 Ryd $(\sim 0.3 \%)$ - see for example, levels 28 and 29. However, the differences for two levels, namely $\left(2 \mathrm{~s}^{2} 2 \mathrm{p}^{5} 4 \mathrm{~d}\right){ }^{3} \mathrm{~F}_{3}^{\circ}$ and ${ }^{1} \mathrm{D}_{2}^{\circ}$ (53 and 56), are unexpectedly large, i.e. 1.4 Ryd or $\sim 1.6 \%$. As stated above, there is no ambiguity in our listings of the identification of the levels. However, a closer examination does reveal that these two levels are well mixed with two other levels, namely ${ }^{1} \mathrm{~F}_{3}^{\circ}$ and ${ }^{3} \mathrm{D}_{2}^{\circ}$ (70 and 69), respectively. To be specific, the mixing coefficients of ${ }^{3} \mathrm{~F}_{3}^{\circ}$ and ${ }^{1} \mathrm{~F}_{3}^{\circ}$ are 0.760 and 0.582 , respectively, and those of ${ }^{1} \mathrm{D}_{2}^{\circ}$ and ${ }^{3} \mathrm{D}_{2}^{\circ}$ are 0.726 and 0.459 , respectively. Therefore, the identification of all these four levels is very clear. Nevertheless, if these two levels ${ }^{3} \mathrm{~F}_{3}^{\circ}$ and ${ }^{1} \mathrm{D}_{2}^{\circ}$ are designated as ${ }^{1} \mathrm{~F}_{3}^{\circ}$ and ${ }^{3} \mathrm{D}_{2}^{\circ}$, respectively, in the NIST data, then there is no discrepancy between theory and experiment. Finally, the energy levels of Zhang \& Sampson (1989) also support our above conclusion, as their results agree with ours within 0.2 Ryd. However, they have probably misindentified the $\left(2 \mathrm{~s}^{2} 2 \mathrm{p}^{5} 3 \mathrm{~s}\right){ }^{1} \mathrm{P}_{1}^{\circ}$ and ${ }^{3} \mathrm{P}_{1}^{\circ}$ levels (3 and 5) in reverse 
Table 2. Comparison of energy levels (in Ryd) of Ni XIX.

\begin{tabular}{|c|c|c|c|c|c|c|c|c|}
\hline Index & Configuration & Level & Expt. & "GRASP & FAC1 & FAC2 & FAC3 & CIV3 \\
\hline 1 & $2 s^{2} 2 p^{6}$ & ${ }^{1} \mathrm{~S}_{0}$ & 0.00000 & 0.00000 & 0.0000 & 0.0000 & 0.0000 & 0.0000 \\
\hline 2 & $2 s^{2} 2 p^{5} 3 s$ & ${ }^{3} \mathrm{P}_{2}^{\circ}$ & 64.74789 & 64.59266 & 64.6260 & 64.6243 & 64.4843 & 64.7487 \\
\hline 3 & $2 s^{2} 2 p^{5} 3 s$ & ${ }^{1} \mathrm{P}_{1}^{\circ}$ & 64.90591 & 64.75556 & 64.7985 & 64.7975 & 64.6398 & 64.9061 \\
\hline 4 & $2 s^{2} 2 p^{5} 3 s$ & ${ }^{3} \mathrm{P}_{0}^{\circ}$ & 66.04590 & 65.89549 & 65.9271 & 65.9254 & 65.7771 & 66.0446 \\
\hline 5 & $2 s^{2} 2 p^{5} 3 s$ & ${ }^{3} \mathrm{P}_{1}^{\circ}$ & 66.14067 & 65.99248 & 66.0313 & 66.0302 & 65.8688 & 66.1407 \\
\hline 6 & $2 s^{2} 2 p^{5} 3 p$ & ${ }^{3} S_{1}^{1}$ & 67.26964 & 67.11863 & 67.1474 & 67.1432 & 67.0258 & 67.2651 \\
\hline 7 & $2 s^{2} 2 p^{5} 3 p$ & ${ }^{3} \mathrm{D}_{2}$ & 67.52411 & 67.38277 & 67.4226 & 67.4217 & 67.2797 & 67.5369 \\
\hline 8 & $2 s^{2} 2 p^{5} 3 p$ & ${ }^{3} \mathrm{D}_{3}$ & 67.72295 & 67.57916 & 67.6142 & 67.6126 & 67.4797 & 67.7241 \\
\hline 9 & $2 s^{2} 2 p^{5} 3 p$ & ${ }^{1} \mathrm{P}_{1}$ & 67.79872 & 67.65968 & 67.6981 & 67.6972 & 67.5554 & 67.8000 \\
\hline 10 & $2 s^{2} 2 p^{5} 3 p$ & ${ }^{3} \mathrm{P}_{2}$ & 67.96467 & 67.82370 & 67.8663 & 67.8659 & 67.7191 & 67.9624 \\
\hline 11 & $2 s^{2} 2 p^{5} 3 p$ & ${ }^{3} \mathrm{P}_{0}$ & 68.48787 & 68.36453 & 68.4100 & 68.4089 & 68.2512 & 68.5097 \\
\hline 12 & $2 s^{2} 2 p^{5} 3 p$ & ${ }^{3} \mathrm{D}_{1}$ & 68.77114 & 68.63561 & 68.6713 & 68.6701 & 68.5236 & 68.7856 \\
\hline 13 & $2 s^{2} 2 p^{5} 3 p$ & ${ }^{3} \mathrm{P}_{1}$ & 69.10029 & 68.95945 & 68.9950 & 68.9937 & 68.8487 & 69.0956 \\
\hline 14 & $2 s^{2} 2 p^{5} 3 p$ & ${ }^{1} D_{2}$ & 69.14025 & 69.00116 & 69.0392 & 69.0383 & 68.8875 & 69.1412 \\
\hline 15 & $2 s^{2} 2 p^{5} 3 p$ & ${ }^{1} \mathrm{~S}_{0}$ & 70.08373 & 70.13098 & 70.2260 & 70.2142 & 69.9528 & 70.1169 \\
\hline 16 & $2 s^{2} 2 p^{5} 3 d$ & ${ }^{3} \mathrm{P}_{0}^{\circ}$ & 71.06029 & 70.91200 & 70.9373 & 70.9311 & 70.7882 & 71.0476 \\
\hline 17 & $2 s^{2} 2 p^{5} 3 d$ & ${ }^{3} \mathrm{P}_{1}^{\circ}$ & 71.14900 & 71.00144 & 71.0272 & 71.0213 & 70.8767 & 71.1404 \\
\hline 18 & $2 s^{2} 2 p^{5} 3 d$ & ${ }^{3} \mathrm{P}_{2}^{\circ}$ & 71.31366 & 71.16951 & 71.1944 & 71.1885 & 71.0422 & 71.3059 \\
\hline 19 & $2 s^{2} 2 p^{5} 3 d$ & ${ }^{3} \mathrm{~F}_{4}^{\circ}$ & 71.30920 & 71.16811 & 71.1954 & 71.1904 & 71.0479 & 71.3149 \\
\hline 20 & $2 s^{2} 2 p^{5} 3 d$ & ${ }^{3} \mathrm{~F}_{3}^{\circ}$ & 71.36068 & 71.22664 & 71.2481 & 71.2433 & 71.0934 & 71.3739 \\
\hline 21 & $2 s^{2} 2 p^{5} 3 d$ & ${ }^{1} \mathrm{D}_{2}^{\circ}$ & 71.50804 & 71.37793 & 71.3982 & 71.3949 & 71.2406 & 71.5139 \\
\hline 22 & $2 s^{2} 2 p^{5} 3 d$ & ${ }^{3} \mathrm{D}_{3}^{\circ}$ & 71.60408 & 71.47633 & 71.4981 & 71.4956 & 71.3360 & 71.6126 \\
\hline 23 & $2 s^{2} 2 p^{5} 3 d$ & ${ }^{3} \mathrm{D}_{1}^{\circ}$ & 72.00285 & 71.91932 & 71.9383 & 71.9363 & 71.7689 & 72.0459 \\
\hline 24 & $2 s^{2} 2 p^{5} 3 d$ & ${ }^{3} \mathrm{~F}_{2}^{\circ}$ & 72.65054 & 72.51867 & 72.5450 & 72.5404 & 72.3843 & 72.6507 \\
\hline 25 & $2 s^{2} 2 p^{5} 3 d$ & ${ }^{3} \mathrm{D}_{2}^{\circ}$ & 72.72649 & 72.59210 & 72.6103 & 72.6068 & 72.4450 & 72.7172 \\
\hline 26 & $2 s^{2} 2 p^{5} 3 d$ & ${ }^{1} \mathrm{~F}_{3}^{\circ}$ & 72.77962 & 72.64864 & 72.6662 & 72.6624 & 72.4993 & 72.7769 \\
\hline 27 & $2 s^{2} 2 p^{5} 3 d$ & ${ }^{1} \mathrm{P}_{1}^{\circ}$ & 73.28227 & 73.24505 & 73.2607 & 73.2589 & 73.0681 & 73.3565 \\
\hline 28 & $2 s^{2} p^{6} 3 s$ & ${ }^{3} S_{1}^{1}$ & 76.16370 & 75.91019 & 75.9615 & 75.9601 & 75.8236 & 74.8222 \\
\hline 29 & $2 s 2 p^{6} 3 s$ & ${ }^{1} \mathrm{~S}_{0}$ & 76.69223 & 76.45810 & 76.5362 & 76.5328 & 76.3398 & 75.3098 \\
\hline 30 & $2 s^{2} p^{6} 3 p$ & ${ }^{3} \mathrm{P}_{0}^{\circ}$ & & 78.62091 & 78.6761 & 78.6750 & 78.5453 & 77.2530 \\
\hline 31 & $2 s^{2} p^{6} 3 p$ & ${ }^{3} \mathrm{P}_{1}^{\circ}$ & 78.56398 & 78.66211 & 78.7185 & 78.7176 & 78.5857 & 77.2996 \\
\hline 32 & $2 s^{2} p^{6} 3 p$ & ${ }^{3} \mathrm{P}_{2}^{\circ}$ & & 78.91529 & 78.9692 & 78.9679 & 78.8400 & 77.5506 \\
\hline 33 & $2 s^{2} p^{6} 3 p$ & ${ }^{1} \mathrm{P}_{1}^{\circ}$ & 78.97314 & 79.06836 & 79.1314 & 79.1314 & 78.9879 & 77.6960 \\
\hline 34 & $2 \mathrm{~s} 2 \mathrm{p}^{6} 3 \mathrm{~d}$ & ${ }^{3} D_{1}$ & & 82.35964 & 82.3940 & 82.3854 & 82.2644 & 80.8726 \\
\hline 35 & $2 s^{2} p^{6} 3 d$ & ${ }^{3} \mathrm{D}_{2}$ & & 82.37523 & 82.4097 & 82.4011 & 82.2800 & 80.8917 \\
\hline 36 & $2 s^{2} p^{6} 3 d$ & ${ }^{3} \mathrm{D}_{3}$ & & 82.40539 & 82.4397 & 82.4311 & 82.3103 & 80.9221 \\
\hline 37 & $2 s^{2} 2 p^{6} 3 d$ & ${ }^{1} \mathrm{D}_{2}$ & & 82.82932 & 82.8588 & 82.8563 & 82.7004 & 81.3372 \\
\hline
\end{tabular}

Expt.: NIST data from http://www . physics.nist.gov/PhysRefData

GRASP: present GRASP results for 89 levels.

FAC1: Present FAC results for 89 levels.

FAC2: Present FAC results for 157 levels.

FAC3: Present FAC results for 3601 levels.

CIV3: Hibbert et al. (1993).

order, as seen in comparison with the experimental work or our calculations in Table 1. Additionally, the energy levels of the NIST compilations and the calculations of Zhang \& Sampson agree comparatively better with our results performed without the inclusion of Breit and QED corrections, listed in Table 1 as GRASP $^{a}$, because Zhang \& Sampson have omitted these in their calculations. The net effect of the Breit and QED corrections is to lower the energies by $\sim 0.11$ Ryd.

The other results listed in Table 1 are from Loulergue \& Nussbaumer (1975), who have included the same configurations as in our GRASP1 calculations, yet their energy levels differ by up to $1.8 \mathrm{Ryd}(\sim 2.5 \%)$ with both experimental and other theoretical results, for many levels, such as: $13,25,28-37$ and 69-81. Some of these differences are due to the misidentification of the levels - see, for example, levels $3 / 5$ and $9 / 13$, but for a majority of the levels (such as 78 and 81) the differences are in the calculational methods. Since their calculations were performed over three decades ago, with limited CI and including only the spinorbit interaction term, it will be unfair to give too much emphasis to these differences in comparison to the present work. However, the energy levels of a more recent calculation by Hibbert et al. (1993) also agree with those of Loulergue \& Nussbaumer, particularly for the levels of the $2 \mathrm{~s} 2 \mathrm{p}^{6} 3 \ell$ configurations (28-37), and are in complete disagreement with the present or earlier calculations of Zhang \& Sampson (1989) - see Table 2. Corresponding results from the experimental work are not available for all the levels, but are available for four levels (28, 29, 31 and 33), for which there is no agreement with the work of Loulergue \& Nussbaumer or Hibbert et al. Since two independent calculations for 10 levels of the $2 \mathrm{~s} 2 \mathrm{p}^{6} 3 \ell$ configurations differ substantially, by up to 1.8 Ryd ( $\sim 2 \%)$, with the present and earlier work of Zhang $\&$ Sampson, we discuss these further.

As stated earlier, Loulergue \& Nussbaumer (1975) included the same basic configurations as included in our GRASP1 calculation or the one performed by Zhang \& Sampson (1989), but Hibbert et al. (1993) included more extensive CI with up to $n=5$ configurations as specified in their Table II. However, the similarity of results between the energy levels of 
Loulergue \& Nussbaumer and Hibbert et al. suggests that inclusion of extensive CI has no effect on the energy levels of the $n=3$ (or even $n=4$ as already discussed) configurations. Nevertheless, to confirm the accuracy of our results we have performed an exercise with the inclusion of extensive CI as included by Hibbert et al. To be specific, we have included 3601 levels arising from the $2 \mathrm{~s}^{2} 2 \mathrm{p}^{6}, 2 \mathrm{~s}^{2} 2 \mathrm{p}^{5} 3 \ell, 2 \mathrm{~s}^{2} 2 \mathrm{p}^{5} 4 \ell, 2 \mathrm{~s}^{2} 2 \mathrm{p}^{5} 5 \ell$, $2 \mathrm{~s} 2 \mathrm{p}^{6} 3 \ell, 2 \mathrm{~s} 2 \mathrm{p}^{6} 4 \ell, 2 \mathrm{~s} 2 \mathrm{p}^{6} 5 \ell, 2 \mathrm{~s} 2 \mathrm{p}^{5} 3 \ell \ell^{\prime}, 2 \mathrm{~s}^{2} 2 \mathrm{p}^{4} 3 \ell \ell^{\prime}, 2 \mathrm{~s} 2 \mathrm{p}^{5} 3 \ell 4 \ell$, $2 \mathrm{~s}^{2} 2 \mathrm{p}^{4} 3 \ell 4 \ell, 2 \mathrm{p}^{6} 3 \ell \ell^{\prime}$, and $2 \mathrm{p}^{6} 3 \ell 4 \ell$ configurations of $\mathrm{Ni}$ XIX. This calculation has been performed with the Flexible Atomic Code (FAC) of $\mathrm{Gu}$ (2003), which is available from the website http://kipac-tree.stanford.edu/fac. This is a fully relativistic code like GRASP, but is comparatively easier to run. Since results obtained from GRASP and FAC are generally in excellent agreement (see, for example, Aggarwal et al. 2007, for $\mathrm{Mg}$-like ions or the present Table 2), our calculations from FAC enable us to assess the contribution of extensive CI in a fully relativistic work. We discuss these results below.

In Table 2, we list energy levels, for the lowest common 37 levels alone, from the experimental compilations of NIST along with a variety of the present calculations, from GRASP as well as FAC, and those of Hibbert et al. (1993). The corresponding energy levels of Zhang \& Sampson (1989) and Loulergue \& Nussbaumer (1975) are not included in this table, because they are similar to the present work and of Hibbert et al, respectively. Additionally, there are 3 calculations from FAC, namely FAC1 (89 levels from the $2 \mathrm{~s}^{2} 2 \mathrm{p}^{6}, 2 \mathrm{~s}^{2} 2 \mathrm{p}^{5} 3 \ell, 2 \mathrm{~s} 2 \mathrm{p}^{6} 3 \ell$, $2 \mathrm{~s}^{2} 2 \mathrm{p}^{5} 4 \ell$ and $2 \mathrm{~s} 2 \mathrm{p}^{6} 4 \ell$ configurations, i.e. the same levels as included in GRASP1), FAC2 (157 levels, i.e. all those of FAC1 plus $2 \mathrm{~s}^{2} 2 \mathrm{p}^{5} 5 \ell$ and $2 \mathrm{~s} 2 \mathrm{p}^{6} 5 \ell$, i.e. the same levels as included in GRASP2), and finally FAC3, which includes 3601 levels arising from the configurations specified above. Our energy levels from GRASP agree closely (within 0.1 Ryd) with those from FAC 1 and FAC2, and the differences are only slightly higher (up to $0.18 \mathrm{Ryd}$ or equivalently $\leq 0.25 \%$ ) with those from FAC 3 . The CIV 3 energy levels of Hibbert et al. for the lowest 27 levels are in excellent agreement with the experimental compilations of NIST. This is because their focus has been mainly on these levels. However, their energy values for the levels of the $2 \mathrm{~s}^{2} \mathrm{p}^{6} 3 \ell$ configurations (28-37) differ from any of our calculations or the compilations of NIST by up to 1.5 Ryd or equivalently $\leq 2 \%$. The excellent agreement among a variety of our calculations, performed with differing amount of CI included and with two independent methods, confirms that the inclusion of extensive CI does not improve the energy levels of the $n=3$ configurations of Ni XIX. Hence, we can state with confidence that the energy levels of Loulergue \& Nussbaumer and Hibbert et al., although agreeing with each other, are not as accurate as reported by us or earlier by Zhang \& Sampson, and are underestimated by up to $1.5 \mathrm{Ryd}$, especially for the levels of the $2 s 2 p^{6} 3 \ell$ configurations. This conclusion is also (partially) supported by the compilation of experimental energy levels by NIST.

\section{Radiative rates}

The absorption oscillator strength $\left(f_{i j}\right)$ and radiative rate $A_{j i}$ (in $\mathrm{s}^{-1}$ ) for a transition $i \rightarrow j$ are related by the following expression:

$f_{i j}=\frac{m c}{8 \pi^{2} e^{2}} \lambda_{j i}{ }^{2} \frac{\omega_{j}}{\omega_{i}} A_{j i}=1.49 \times 10^{-16} \lambda_{j i}^{2}\left(\omega_{j} / \omega_{i}\right) A_{j i}$

where $m$ and $e$ are the electron mass and charge, respectively, $c$ is the velocity of light, $\lambda_{j i}$ is the transition energy/wavelength in $\AA$, and $\omega_{i}$ and $\omega_{j}$ are the statistical weights of the lower $(i)$ and upper $(j)$ levels, respectively. Similarly, the oscillator strength $f_{i j}$ (dimensionless) and the line strength $S$ (in atomic unit, $1 \mathrm{au}=$ $6.460 \times 10^{-36} \mathrm{~cm}^{2} \mathrm{esu}^{2}$ ) are related by the following standard equations:

for the electric dipole (E1) transitions:

$A_{j i}=\frac{2.0261 \times 10^{18}}{\omega_{j} \lambda_{j i}^{3}} S^{E 1} \quad$ and $\quad f_{i j}=\frac{303.75}{\lambda_{j i} \omega_{i}} S^{E 1}$,

for the magnetic dipole (M1) transitions:

$A_{j i}=\frac{2.6974 \times 10^{13}}{\omega_{j} \lambda_{j i}^{3}} S^{M 1} \quad$ and $\quad f_{i j}=\frac{4.044 \times 10^{-3}}{\lambda_{j i} \omega_{i}} S^{M 1}$,

for the electric quadrupole (E2) transitions:

$A_{j i}=\frac{1.1199 \times 10^{18}}{\omega_{j} \lambda_{j i}^{5}} S^{E 2} \quad$ and $\quad f_{i j}=\frac{167.89}{\lambda_{j i}^{3} \omega_{i}} S^{E 2}$,

and for the magnetic quadrupole (M2) transitions:

$A_{j i}=\frac{1.4910 \times 10^{13}}{\omega_{j} \lambda_{j i}^{5}} S^{M 2} \quad$ and $\quad f_{i j}=\frac{2.236 \times 10^{-3}}{\lambda_{j i}^{3} \omega_{i}} S^{M 2}$

In Table 3 we present transition energies $\left(\Delta E_{i j}\right.$ in $\AA$ ), radiative rates $\left(A_{j i}\right.$ in s $\left.{ }^{-1}\right)$, oscillator strengths ( $f_{i j}$, dimensionless), and line strengths $(S$ in au), in length form only, for all 1210 electric dipole (E1) and 1468 electric quadrupole (E2) transitions among the 89 levels of Ni XIX. The indices used to represent the lower and upper levels of a transition have already been defined in Table 1. Similar results for 1172 magnetic dipole (M1) and 1505 magnetic quadrupole (M2) transitions are listed in Table 4.

Since the electric dipole (E1) transitions are comparatively more important, and most of the results available in the literature are confined to these transitions alone, we focus on the accuracy assessments of their $f$-values. In Table 5 we compare our results from the GRASP1, GRASP2 and FAC calculations with those of Hibbert et al. (1993) from the CIV3 code. The FAC calculations correspond to the FAC3 configurations described in Sect. 2, and the corresponding results of Zhang \& Sampson (1989) are not included in this table, because their $f$-values are only for the resonance transitions, for which there is no discrepancy with our calculations. Furthermore, in this table we restrict the comparisons to transitions from the lowest 5 levels to levels up to 37 . This comparison should give us sufficient information about the accuracy of our radiative rates.

Our $f$-values from the GRASP1, GRASP2 and FAC calculations generally agree within $\sim 10 \%$ for all the transitions listed in Table 5 . This highly satisfactory agreement among the three calculations, with differing amount of CI included, clearly shows that the effect of extensive CI is negligible on the $f$-values. Similarly, the earlier obtained results of Hibbert et al. (1993) from the CIV3 code also generally agree within $10 \%$ for a majority of transitions. However, there are differences of up to $20 \%$ for some transitions, such as: 2-28, 3-28 and 4-28, whereas for the $5-29\left(2 \mathrm{~s}^{2} 2 \mathrm{p}^{5} 3 \mathrm{~s}^{3} \mathrm{P}_{1}^{\circ}-2 \mathrm{~s} 2 \mathrm{p}^{6} 3 \mathrm{~s}^{1} \mathrm{~S}_{0}\right)$ transition their $f$-value is lower by $50 \%$ in comparison to all three other results listed in the table. Since all the transitions for which the $f$-values of Hibbert et al. differ substantially, including those which are not listed in Table 5, involve the higher excited levels of the $2 \mathrm{~s}^{2} \mathrm{p}^{6} 3 \ell$ configurations (28-37), the differences are clearly due to the inaccuracy in their determination of energy levels, as already discussed 
Table 5. Comparison of oscillator strengths for some transitions of Ni XIX. $\left(a \pm b \equiv a \times 10^{ \pm b}\right)$.

\begin{tabular}{rrllll}
\hline \hline \multicolumn{1}{l}{ Transition } & GRASP1 & GRASP2 & FAC & CIV3 \\
\hline 1 & 3 & $1.300-1$ & $1.2931-1$ & $1.252-1$ & $1.254-1$ \\
1 & 5 & $9.927-2$ & $9.8688-2$ & $9.382-2$ & $9.420-2$ \\
1 & 17 & $1.025-2$ & $1.0273-2$ & $9.979-3$ & $1.130-2$ \\
1 & 23 & $8.188-1$ & $8.1883-1$ & $8.201-1$ & $7.986-1$ \\
1 & 27 & $2.457-0$ & $2.4206-0$ & $2.287-0$ & $2.300-0$ \\
1 & 31 & $4.709-2$ & $4.6852-2$ & $4.876-2$ & $4.250-2$ \\
1 & 33 & $2.898-1$ & $2.8881-1$ & $2.894-1$ & $2.767-1$ \\
2 & 6 & $4.957-2$ & $4.9457-2$ & $4.967-2$ & $4.854-2$ \\
2 & 7 & $4.855-2$ & $4.8515-2$ & $4.733-2$ & $4.742-2$ \\
2 & 8 & $1.548-1$ & $1.5469-1$ & $1.531-1$ & $1.522-1$ \\
2 & 9 & $1.873-3$ & $1.8944-3$ & $1.662-3$ & $1.940-3$ \\
2 & 10 & $6.557-2$ & $6.5560-2$ & $6.481-2$ & $6.476-2$ \\
2 & 12 & $1.128-4$ & $1.1365-4$ & $1.142-4$ & $1.200-4$ \\
2 & 13 & $3.868-3$ & $3.8913-3$ & $3.587-3$ & $3.940-3$ \\
2 & 14 & $7.111-4$ & $7.1384-4$ & $6.739-4$ & $7.400-4$ \\
2 & 28 & $6.660-2$ & $6.6925-2$ & $6.192-2$ & $5.336-2$ \\
3 & 6 & $1.500-3$ & $1.5137-3$ & $1.415-3$ & $1.567-3$ \\
3 & 7 & $8.620-2$ & $8.6214-2$ & $8.591-2$ & $8.580-2$ \\
3 & 9 & $1.038-1$ & $1.0380-1$ & $1.026-1$ & $1.015-1$ \\
3 & 10 & $9.025-2$ & $9.0265-2$ & $8.797-2$ & $8.757-2$ \\
3 & 11 & $3.395-2$ & $3.4100-2$ & $3.356-2$ & $3.440-2$ \\
3 & 12 & $3.303-4$ & $3.3966-4$ & $3.079-4$ & $3.333-4$ \\
3 & 13 & $7.318-5$ & $7.7306-5$ & $6.551-5$ & $6.667-5$ \\
3 & 14 & $8.679-4$ & $8.5801-4$ & $8.228-4$ & $8.333-4$ \\
3 & 15 & $2.360-2$ & $2.3411-2$ & $2.247-2$ & $2.157-2$ \\
3 & 28 & $2.867-2$ & $2.8813-2$ & $2.641-2$ & $2.310-2$ \\
3 & 29 & $2.568-2$ & $2.5681-2$ & $2.409-2$ & $1.890-2$ \\
4 & 6 & $1.157-3$ & $1.1556-3$ & $1.216-3$ & $1.200-3$ \\
4 & 9 & $2.027-4$ & $2.0351-4$ & $1.915-4$ & $2.000-4$ \\
4 & 12 & $1.130-1$ & $1.1288-1$ & $1.103-1$ & $1.109-1$ \\
4 & 13 & $2.082-1$ & $2.0805-1$ & $2.069-1$ & $2.048-1$ \\
4 & 28 & $6.083-2$ & $6.1118-2$ & $5.653-2$ & $4.950-2$ \\
5 & 6 & $5.057-4$ & $5.0582-4$ & $5.352-4$ & $5.000-4$ \\
5 & 9 & $3.113-5$ & $2.9827-5$ & $4.039-5$ & $3.333-5$ \\
5 & 10 & $7.700-4$ & $7.7368-4$ & $7.448-4$ & $7.333-4$ \\
5 & 11 & $8.211-3$ & $8.1051-3$ & $7.902-3$ & $7.500-3$ \\
5 & 12 & $5.982-2$ & $5.9843-2$ & $5.948-2$ & $5.920-1$ \\
5 & 13 & $3.826-2$ & $3.8248-2$ & $3.743-2$ & $3.707-2$ \\
5 & 14 & $1.867-1$ & $1.8663-1$ & $1.841-1$ & $1.833-1$ \\
5 & 15 & $4.149-2$ & $4.1547-2$ & $4.034-2$ & $4.073-2$ \\
5 & 28 & $3.397-2$ & $3.4138-2$ & $3.184-2$ & $2.757-2$ \\
5 & 29 & $1.645-2$ & $1.6461-2$ & $1.513-2$ & $1.177-2$ \\
5 & 37 & $2.197-4$ & $2.2982-4$ & $2.493-4$ & $2.000-4$ \\
\hline
\end{tabular}

GRASP1: present calculations from the GRASP code with 89 levels. GRASP2: present calculations from the GRASP code with 157 levels. FAC: present calculations from the FAC code with 3601 levels. CIV3: calculations of Hibbert et al. (1993) from the CIV3 code.

in Sect. 2. Therefore, there is no discrepancy with the reported $f$-values of Hibbert et al. for transitions involving lower levels up to 27 , but their results for those transitions which involve the higher excited levels of the $2 \mathrm{~s} 2 \mathrm{p}^{6} 3 \ell$ configurations are not very accurate, and differ from our calculations by up to $50 \%$. However, before forming conclusions on the accuracy of our results, we make some other assessments below for a larger number of transitions, because the transitions in Table 5 represent only a fraction of a total of 1210 .

A general criterion to assess the accuracy of the $A$ - or $f$-values is to compare their length and velocity forms. Before we discuss these we would like to remind readers that such comparisons are desirable, but are not a fully sufficient tests to assess the accuracies (Hibbert 1975b), as different calculations (or combinations of configurations) may give comparable $f$-values in the two forms, but entirely different results in magnitude see Aggarwal et al. (2007) for further details and comparisons. Nevertheless, we discuss the two forms below in order to make some assessment about the accuracy of the results.

Among the stronger transitions (i.e. $f \geq 0.01$ ), the length and velocity forms agree to better than $20 \%$ for all transitions, except for 11-27 $\left(2 \mathrm{~s}^{2} 2 \mathrm{p}^{5} 3 \mathrm{p}^{3} \mathrm{P}_{0}-2 \mathrm{~s}^{2} 2 \mathrm{p}^{5} 3 \mathrm{~d}^{1} \mathrm{P}_{1}^{\circ} ; f_{L}=0.017\right)$ for which $f_{L} / f_{V}=1.5$. This is highly satisfactory and in a way confirms the accuracy of our calculations. However, differences between the two forms for weaker transitions $(f<0.01)$ are up to several orders of magnitude, particularly for those whose $f$-values are $\sim 10^{-5}$ or smaller. Examples of such transitions are: 7-80 $\left(f=1.3 \times 10^{-5}\right), 14-81\left(f=1.5 \times 10^{-5}\right)$ and 52-55 $\left(f=9.2 \times 10^{-8}\right)$. Such large differences for weak transitions are very common, and mainly arise from the cancellation effect of the mixing coefficients and/or the inclusion/exclusion of large CI. Although the $f$ - or $A$-values for such transitions may be required in modelling work, their contribution is much less important in comparison to those from the stronger transitions. Therefore, a larger variation in their values among different calculations, and/or between their $f_{L}$ and $f_{V}$ values, do not affect the overall accuracy of a calculation. To conclude, based on a satisfactory agreement between the two forms, we may state that the accuracy of our listed $A$-values is better than $20 \%$ for all strong transitions.

\section{Lifetimes}

The lifetime $\tau$ for a level $j$ is defined as follows:

$\tau_{j}=\frac{1}{\sum_{i} A_{j i}}$.

Since this is a measurable parameter, it provides a check on the accuracy of the calculations. In Table 1 we list our calculated lifetimes, from both the GRASP1 and GRASP 2 calculations, which include the contributions from all four types of transitions, i.e. E1, E2, M1 and M2. These results are helpful for comparisons with theory or experiments. The two sets of lifetimes agree closely, except for two levels, namely $\left(2 \mathrm{~s} 2 \mathrm{p}^{6} 4 \mathrm{p}\right){ }^{3} \mathrm{P}_{1}^{\circ}$ and ${ }^{1} \mathrm{P}_{1}^{\circ}$ (79 and 81), for which the differences are up to a factor of two. However, the lifetimes for both of these levels are very small, i.e. $\tau \sim 10^{-13} \mathrm{~s}$.

To our knowledge, no measurements of lifetimes are available for levels of Ni XIX, but Hibbert et al. (1993) have reported calculations for some levels of the $2 \mathrm{~s}^{2} 2 \mathrm{p}^{5} 3 \ell$ configurations. We compare their results with our calculations in Table 6 . In general, agreement between the two calculations is within $10 \%$, which is highly satisfactory. However, the difference for the $2 s^{2} 2 p^{5} 3 p^{1} D_{2}$ level (14) is $\sim 20 \%$, which is due to the fact that Hibbert et al. have performed calculations for the E1 transitions alone, whereas we have also included the contributions of the E2, M1 and M2 transitions. For most of the levels listed in Table 6 the contributions of the E1 transitions are dominant, but for the ${ }^{1} \mathrm{D}_{2}$ level, the E2 transition 1-14 makes a significant contribution, as its $A$-value is $1.20 \times 10^{9} \mathrm{~s}^{-1}$ while that of the dominant 5-14 E1 transition is $8.14 \times 10^{9} \mathrm{~s}^{-1}$. To conclude, we may state that there is no discrepancy for the lifetimes between two independent calculations, because for the lowest 27 levels there is no discrepancy for the $A$-values either.

\section{Conclusions}

In the present work, results for energy levels, radiative rates, oscillator strengths, and line strengths for transitions among the 
Table 6. Comparison of lifetimes ( $\tau$ in ns) for some levels of Ni XIX. $a \pm b \equiv a \times 10^{ \pm b}$.

\begin{tabular}{rlcl}
\hline \hline Index & Level & Present Calculations & Hibbert et al. (1993) \\
\hline 6 & $2 \mathrm{~s}^{2} 2 \mathrm{p}^{5} 3 \mathrm{p}^{3} \mathrm{~S}_{1}$ & $2.319-1$ & $2.38-1$ \\
7 & $2 \mathrm{~s}^{2} 2 \mathrm{p}^{5} 3 \mathrm{p}^{3} \mathrm{D}_{2}$ & $1.456-1$ & $1.72-1$ \\
8 & $2 \mathrm{~s}^{2} 2 \mathrm{p}^{5} 3 \mathrm{p}^{3} \mathrm{D}_{3}$ & $1.262-1$ & $1.29-1$ \\
9 & $2 \mathrm{~s}^{2} 2 \mathrm{p}^{5} 3 \mathrm{p}^{1} \mathrm{P}_{1}$ & $1.376-1$ & $1.41-1$ \\
10 & $2 \mathrm{~s}^{2} 2 \mathrm{p}^{5} 3 \mathrm{p}^{3} \mathrm{P}_{2}$ & $9.417-2$ & $1.07-1$ \\
11 & $2 \mathrm{~s}^{2} 2 \mathrm{p}^{5} 3 \mathrm{p}^{3} \mathrm{P}_{0}$ & $8.498-2$ & $8.49-2$ \\
12 & $2 \mathrm{~s}^{2} 2 \mathrm{p}^{5} 3 \mathrm{p}^{3} \mathrm{D}_{1}$ & $1.757-1$ & $1.78-1$ \\
13 & $2 \mathrm{~s}^{2} 2 \mathrm{p}^{5} 3 \mathrm{p}^{3} \mathrm{P}_{1}$ & $1.119-1$ & $1.15-1$ \\
14 & $2 \mathrm{~s}^{2} 2 \mathrm{p}^{5} 3 \mathrm{p}^{1} \mathrm{D}_{2}$ & $1.049-1$ & $1.23-1$ \\
15 & $2 \mathrm{~s}^{2} 2 \mathrm{p}^{5} 3 \mathrm{p}^{1} \mathrm{~S}_{0}$ & $2.980-2$ & $3.37-2$ \\
& & & \\
3 & $2 \mathrm{~s}^{2} 2 \mathrm{p}^{5} 3 \mathrm{~s}^{1} \mathrm{P}_{1}^{\circ}$ & $6.851-4$ & $7.07-4$ \\
5 & $2 \mathrm{~s}^{2} 2 \mathrm{p}^{5} 3 \mathrm{~s}^{3} \mathrm{P}_{1}^{\circ}$ & $8.639-4$ & $9.07-4$ \\
16 & $2 \mathrm{~s}^{2} 2 \mathrm{p}^{5} 3 \mathrm{~d}^{3} \mathrm{P}_{0}^{\circ}$ & $7.318-2$ & $7.57-2$ \\
17 & $2 \mathrm{~s}^{2} 2 \mathrm{p}^{5} 3 \mathrm{~d}^{3} \mathrm{P}_{1}^{\circ}$ & $6.579-3$ & $6.03-3$ \\
18 & $2 \mathrm{~s}^{2} 2 \mathrm{p}^{5} 3 \mathrm{~d}^{3} \mathrm{P}_{2}^{\circ}$ & $7.524-2$ & $7.76-2$ \\
19 & $2 \mathrm{~s}^{2} 2 \mathrm{p}^{5} 3 \mathrm{~d}^{3} \mathrm{~F}_{4}^{\circ}$ & $7.866-2$ & $8.00-2$ \\
20 & $2 \mathrm{~s}^{2} 2 \mathrm{p}^{5} 3 \mathrm{~d}^{3} \mathrm{~F}_{3}^{\circ}$ & $6.555-2$ & $6.69-2$ \\
21 & $2 \mathrm{~s}^{2} 2 \mathrm{p}^{5} 3 \mathrm{~d}^{1} \mathrm{D}_{2}^{\circ}$ & $6.317-2$ & $6.46-2$ \\
22 & $2 \mathrm{~s}^{2} 2 \mathrm{p}^{5} 3 \mathrm{~d}^{3} \mathrm{D}_{3}^{\circ}$ & $6.954-2$ & $7.08-2$ \\
23 & $2 \mathrm{~s}^{2} 2 \mathrm{p}^{5} 3 \mathrm{~d}^{3} \mathrm{D}_{1}^{\circ}$ & $8.806-5$ & $9.00-5$ \\
24 & $2 \mathrm{~s}^{2} 2 \mathrm{p}^{5} 3 \mathrm{~d}^{3} \mathrm{~F}_{2}^{\circ}$ & $6.377-2$ & $6.58-2$ \\
25 & $2 \mathrm{~s}^{2} 2 \mathrm{p}^{5} 3 \mathrm{~d}^{3} \mathrm{D}_{2}^{\circ}$ & $7.158-2$ & $7.35-2$ \\
26 & $2 \mathrm{~s}^{2} 2 \mathrm{p}^{5} 3 \mathrm{~d}^{1} \mathrm{~F}_{3}^{\circ}$ & $7.330-2$ & $7.52-2$ \\
27 & $2 \mathrm{~s}^{2} 2 \mathrm{p}^{5} 3 \mathrm{~d}^{1} \mathrm{P}_{1}^{\circ}$ & $2.832-5$ & $3.02-5$ \\
\hline
\end{tabular}

89 levels of Ni XIX have been presented for all permissible transitions. These results cover a substantially larger number of transitions than previously available in the literature. Additionally, results for radiative rates have been presented for four types of transitions, namely E1, E2, M1 and M2. A complete set of results are likely to be useful for the modelling of a variety of plasmas. Furthermore, calculations for $A$-values have also been performed for a larger number of transitions among 157 levels, which include additional 68 levels of the $2 \mathrm{~s}^{2} 2 \mathrm{p}^{5} 5 \ell$ and $2 \mathrm{~s}^{2} \mathrm{p}^{6} 5 \ell$ configurations. This entire set of data for $3401 \mathrm{E} 1,4439 \mathrm{E} 2$, $3334 \mathrm{M} 1$ and $4503 \mathrm{M} 2$ transitions can be obtained in electronic form on request from KMA (K. Aggarwal@qub. ac.uk).

Based on the comparison made among a variety of calculations, and adopting both the GRASP and FAC codes with differing amount of CI, as well as with the available compiled experimental and other theoretical results, our energy levels are assessed to be accurate to better than $1 \%$. We have also demonstrated that the earlier available energy levels of Loulergue \& Nussbaumer (1975) and Hibbert et al. (1993) are underestimated by up to 1.5 Ryd for several levels, particularly the higher ones. Similarly, based on the comparison made between the length and velocity forms of the oscillator strengths, as well as among a variety of calculations with differing amount of CI included, we assess that our radiative rates are accurate to better than $20 \%$ for all strong transitions. However, for weaker transitions the $A$ - or $f$-values are less accurate.

Lifetimes for all excited levels of Ni XIX are listed, but comparison with the corresponding available results has been possible for only a few levels, for which there are no discrepancies.

To assess the accuracy of our presented results, we also performed a larger calculation (with up to 3601 levels) from FAC, which included more elaborate CI than in our GRASP calculations. However, the energy levels and $A$-values from GRASP have been preferred, mainly for two reasons. Firstly, as discussed in Sects. 2 and 3, the results obtained from the simpler GRASP calculations are of comparable accuracy with those from the larger calculation. Secondly, and more importantly, a collisional calculation for 3601 levels is currently not feasible with the resources available to us. Therefore, our calculations for a more important parameter namely collision strength, and subsequently for excitation rate coefficient, are in progress only for the lowest 89 levels of Ni XIX. Thus the presently reported results will be consistent with the collisional work. Nevertheless, energy levels and radiative rates for all the 3601 levels can be obtained in electronic form on request from KMA (K.Aggarwal@qub.ac.uk).

Acknowledgements. This work has been financed by the Engineering and Physical Sciences and Particle Physics and Astronomy Research Councils of the United Kingdom, and F.P.K. is grateful to AWE Aldermaston for the award of a William Penney Fellowship.

\section{References}

Aggarwal, K. M., Keenan, F. P., \& Msezane, A. Z. 2003, At. Data Nucl. Data Tables, 85, 453

Aggarwal, K. M., Tayal, V., Gupta, G. P., \& Keenan, F. P. 2007, At. Data Nucl. Data Tables, in press

Biémont, E., et al. 2000, J. Phys. B, 33, 2153

Boiko, V. A., Pikuz, S. A., Safronova, U. I., \& Faenov, A. Ya 1977, J. Phys. B, 10,1253

Buchet, J. P., Buchet-Poulizac, M. C., Denis, A., et al. 1987, J. Phys. B, 20, 1709 Del Zanna, G., Berrington, K. A., \& Mason, H. E. 2004, A\&A, 422, 731

Dyall, K. G., Grant, I. P., Johnson, C. T., Parpia, F. A., \& Plummer, E. P. 1989, Comput. Phys. Commun., 55, 424

Feldman, U., Cohan, L., \& Swartz, M. 1967, ApJ, 148, 585

Feldman, U., Curdt, W., Landi, E., \& Wilhelm, K. 2000, ApJ, 544, 508

Gu, M. F. 2003, ApJ, 582, 1241

Hibbert, A. 1975a, Comput. Phys. Commun., 9, 141

Hibbert, A. 1975b, Rep. Prog. Phys., 38, 1217

Hibbert, A., Le Dourneuf, M., \& Mohan, M. 1993, At. Data Nucl. Data Tables, 53,23

Jupen, C. 1984, MNRAS, 208, 1P

Liedahl, D. A. 2000, Atomic Data Needs for X-ray Astronomy available at http://heasarc.gsfc.nasa.gov/docs/heasarc/atomic/

Loulergue, M., \& Nussbaumer, H. 1975, A\&A, 45, 125

Swartz, M., Kastner, S., Rothe, E., \& Neupert, W. 1971, J. Phys. B, 4, 1747

Zhang, H. L., Sampson, D. H., Clark, R. E. H., \& Mann, J. B. 1987, At. Data Nucl. Data Tables, 37, 17

Zhang, H. L., \& Sampson, D. H. 1989, At. Data Nucl. Data Tables, 43, 1 